\title{
Brexit: Unsicherheit ist Gift für die Wirtschaft
}

\section{Till Nikolaus Folger}

\section{Relevanz}

Kommt es zu einem harten Brexit? Müssen sich die Unternehmen auf neue Zölle, kostspielige Zollformalitäten, Zeitverzögerungen an der Grenze, teure Unterbrüche in der Wertschöpfungskette, erhöhten Kapitalbedarf für die Lagerhaltung, unterschiedliche Produktstandards, abweichende Rechtsvorschriften, und weiter zunehmende Kosten einstellen? Zahlen sich für viele kleinere Unternehmen die Geschäfte mit der EU überhaupt noch aus? Oder gibt es am Ende doch noch ein kooperatives Ergebnis mit beidseitigem Marktzugang zu einem gemeinsamen, einheitlichen Binnenmarkt? Sie wissen es nicht, müssen für alle Eventualitäten planen, und schieben ihre Entscheidungen hinaus. Unsicherheit ist Gift für die Wirtschaft. Bevor überhaupt eine Entscheidung gefallen ist, bremst die BrexitUnsicherheit die britischen Unternehmen und beeinträchtigt Investitionen und Produktivitätswachstum.

\section{Quelle}

Bloom, Nicholas, Philip Bunn, Scarlet Chen, Paul Mizen, Pawel Smietanka, and Gregory Thwaites (2019), The Impact of Brexit on UK Firms, NBER WP 26.218.

Kaum ein Thema hat die europäische Debatte in Politik und Wirtschaft in den letzten Jahren so geprägt wie die Entscheidung der Briten vom Juni 2016, die EU zu verlassen. Während viele Ereignisse in den vergangenen Jahrzehnten zeit-

T. N. Folger $(\bowtie)$

Universität St.Gallen, St.Gallen, Schweiz

E-Mail: till.folger2@student.unisg.ch 
weise für Unsicherheit in der Volkswirtschaft gesorgt haben - man denke an die Golfkriege, die Terroranschläge vom 11. September 2001 oder den Kollaps von Lehman Brothers - war in den meisten Fällen ebenjene Unsicherheit mit dem Aufkommen neuer Informationen schnell wieder verflogen. Anders verhält es sich beim Brexit. Mehr als drei Jahre nach dem Referendum bleibt die Zukunft des Vereinigten Königreichs und seiner Beziehung zur EU weiter völlig unklar. Für die Unternehmen sind eine Reihe relevanter Fragestellungen bisher ungeklärt: Wie sieht der Zugang zum EU-Binnenmarkt aus? Wie viele ausländische Fachkräfte können weiterhin angeworben werden? Wie werden britische Produktvorschriften genau aussehen? Genauso wenig wie die zukünftige Position des Vereinigten Königreichs ist der Übergang dahin bisher definiert.

Vor allem im Land selbst wird weiterhin erbittert diskutiert. Ökonomen versuchen durch Analysen und Prognosen, Orientierung über die Zukunft zu geben. Dabei werden aber oft die bereits jetzt sichtbaren wirtschaftlichen Auswirkungen des Brexits seit dem Referendum übersehen. Während die künftigen wirtschaftlichen Rahmenbedingungen der britischen Wirtschaft höchst ungewiss bleiben, kann man bereits jetzt die Auswirkungen der anhaltenden Unsicherheit in den bestehenden Daten feststellen.

Wie sich die Ungewissheit wirtschaftlich ausgewirkt hat, haben sechs Volkswirte um Nicholas Bloom von der amerikanischen Universität Stanford in einer im September erschienen Forschungsarbeit untersucht. Indem sie nicht über verschiedene Zukunftsszenarien spekulieren, sondern die tatsächlich sichtbaren Effekte der Unsicherheit der letzten drei Jahre untersuchen, rücken sie die Debatte um den Brexit in ein neues Licht.

Als Basis ihrer Arbeit diente den Forschern das „Decision Maker Panel“ (DMP), mit welchem sie seit August 2016 monatlich Daten tausender Unternehmen erheben. Von allen $42^{\prime} 000$ britischen Unternehmen mit mindestens zehn Mitarbeitern haben sie eine zufällige Auswahl von Unternehmen eingeladen, an einer Online-Befragung teilzunehmen. Insgesamt haben 5'900 Unternehmen in den drei untersuchten Jahren mindestens eine Frage zum Thema Brexit im DMP beantwortet. Mit total rund 3.7 Mio. Beschäftigten repräsentieren diese etwa $14 \%$ der britischen Arbeitsplätze im Privatsektor. Die Fragen wurden meist durch die Spitzenmanager der jeweiligen Unternehmen beantwortet: Bei $70 \%$ war dies der Finanzvorstand, bei $15 \%$ sogar der Vorstandsvorsitzende.

Eine Schlüsselfrage des DMP-Panels lautet: „Wie sehr hat das Ergebnis des EU-Referendums den Grad an Unsicherheit in Ihrem Unternehmen beeinflusst?" Aus den vier Antwortmöglichkeiten leiteten die Autoren den BrexitUnsicherheits-Index (BUI) ab. Dieser zeigt den Anteil jener Unternehmen, welche den Brexit als den wichtigsten oder einen der drei wichtigsten Unsicherheitsfaktoren bezeichnen. Betrachteten unmittelbar nach dem Referendum noch 


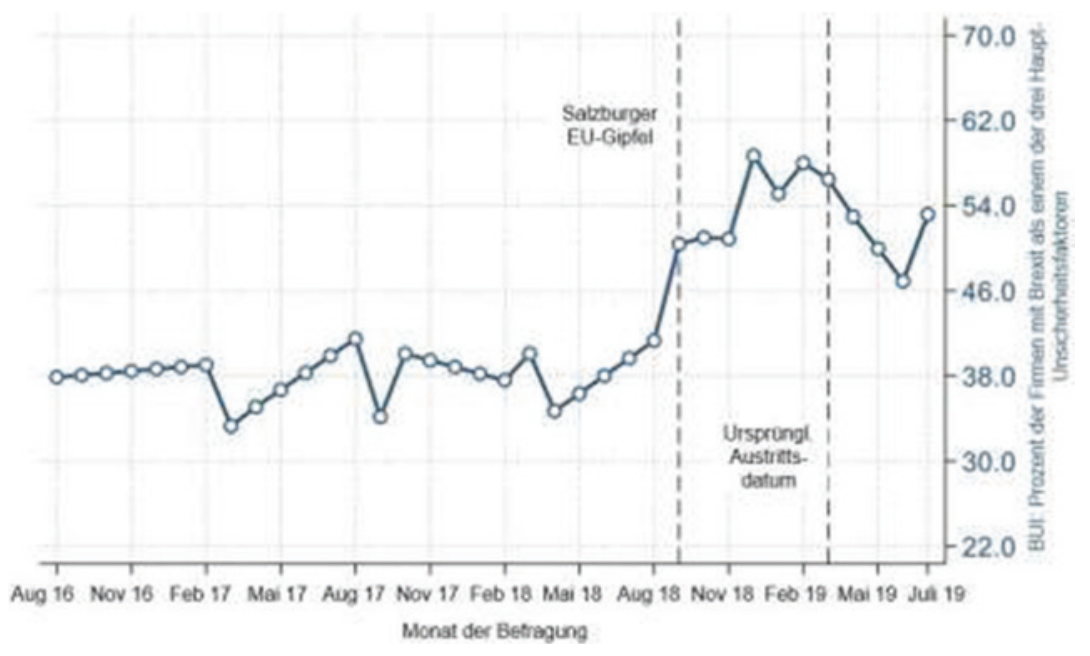

Abb. 1 Der Brexit-Unsicherheits-Index. (Quelle: Bloom u. a. 2019, Abb.1)

weniger als $40 \%$ den Brexit als einen der wichtigsten Unsicherheitsfaktoren an, waren es zweieinhalb Jahre später im Juli 2019 deutlich über die Hälfte. Abb. 1 zeigt, dass die Unsicherheit weiter anhält und jüngst stark zugenommen hat.

Viele verschiedene Faktoren zeichneten für die Unsicherheit durch den Brexit verantwortlich. Die Unternehmen nannten hierbei vor allem Einflüsse auf Arbeitsmarkt, Regulierung, Produktnachfrage, Zölle und Lieferketten. Während der BUI seit dem Referendum gestiegen ist, gilt dies nicht für andere Indikatoren, die üblicherweise zur Messung von Unsicherheit zu Rate gezogen werden. So schlug zwar der britische Aktienmarkt als direkte Reaktion auf das Votum deutlich nach unten aus, normalisierte sich jedoch innerhalb weniger Wochen wieder. Nicholas Bloom und seine Kollegen liefern somit erstmals ein Mass, welches die Unsicherheit im britischen Geschäftsumfeld langfristig erfassen kann.

Der Brexit-Unsicherheits-Index BUI zeigt, dass die Unsicherheit auch drei Jahre nach dem Referendum anhält und aktuell stark zugenommen hat. Mittlerweile betrachten mehr Unternehmen den Brexit als einen der drei grössten Unsicherheitsfaktoren als noch 2016.

Im zweiten Teil der Studie gingen die Forscher der Frage nach, wie die Unsicherheit über den Brexit das Verhalten der betroffenen Unternehmen und damit Investitionen und Produktivität beeinflusst. Ihre Analyse ergab, dass Unternehmen mit engeren Verbindungen zur EU etwa durch grössere Exporte in den 
Rest der EU oder einen höheren Anteil von Arbeitnehmern aus anderen EU-Mitgliedsstaaten die Unsicherheit aufgrund des Brexits signifikant höher einschätzen.

Die empirischen Schätzungen zeigen, dass das Investitionswachstum eines Unternehmens seit dem Referendum um 2.8 Prozentpunkte geringer ausfiel, wenn es die Unsicherheit durch den Brexit um einen Punkt auf der vierteiligen Skala höher einschätzte. Auf Basis dieser Ergebnisse kamen die Autoren zum Schluss, dass die gesamten Investitionen über drei Jahre rund $11 \%$ niedriger waren als sie es ohne den Brexit und die dadurch entstandene Unsicherheit gewesen wären. Entgegen der Erwartung eines abrupten Investitionsstopps nach dem Referendum und einer darauffolgenden Erholung kam es tatsächlich zu einem schleichenden, aber anhaltenden Rückgang über den Gesamtzeitraum.

Durch den Brexit haben die britischen Unternehmen ihre Investitionen seit 2016 um total 11 Prozent zurückgefahren. Der stetige Rückgang unterstreicht die tiefgreifende Verunsicherung der britischen Wirtschaft.

Ausserdem zeigt das Forscherteam, dass der Brexit merkliche Produktivitätseinbussen verursacht. Eine Hauptursache liegt darin, dass die Planungen für den Brexit wertvolle Ressourcen im Unternehmen in Anspruch nehmen. So gaben beispielsweise rund $70 \%$ der befragten Unternehmen an, dass ihre Manager mehrere Stunden wöchentlich mit entsprechenden Planungen befasst waren. Finanziell beliefen sich die Aufwendungen hierfür im Schnitt auf $0.4 \%$ des jeweiligen Umsatzes.

Das jährliche Produktivitätswachstum in einem Unternehmen fiel um bis zu 1.5 Prozentpunkte niedriger aus, wenn dieses die Brexit-Unsicherheit um einen Punkt auf der vierteiligen Skala - also um ein Viertel - höher einschätzte. Daraus berechnen die Forscher, dass das Produktivitätswachstum über die drei Jahre um zwischen 1.8 und 4.5 Prozentpunkte geringer war als es ohne Brexit der Fall gewesen wäre.

Zudem betrifft der Brexit produktive und wenig produktive Unternehmen in unterschiedlicher Weise: Gerade besonders produktive Unternehmen waren etwa durch Handel stark mit dem EU-Binnenmarkt verflochten und leiden stark unter der derzeitigen Unsicherheit. Die Folge ist eine Umverteilung wirtschaftlicher Aktivität von produktiven, international aufgestellten Unternehmen zu weniger produktiven, lokalen Firmen, was das Produktivitätswachstum weiter verlangsamt. Insgesamt schätzen die Forscher die gesamten Produktivitätseinbussen seit 2016 unter Berücksichtigung dieses Effekts auf zwischen 2 und 5 Prozentpunkte. 
Aufgrund der Unsicherheit ist seit dem Referendum die Produktivität im Vereinigten Königreich um insgesamt 2 bis 5 Prozentpunkte zurückgegangen. Ein Teil dieses Rückgangs ist auf den hohen Aufwand des Managements für Zukunftsplanung zurückzuführen.

Wie der Brexit letzten Endes ausgehen wird, bleibt weiter unklar. Damit bleiben die Konsequenzen für die britische Volkswirtschaft und ihre Beschäftigten ebenfalls unklar. Dank Bloom und seinem Forscherteam wissen wir allerdings, dass die anhaltende Unsicherheit der letzten drei Jahre bereits jetzt für signifikant geringere Investitionen und merkliche Produktivitätseinbussen gesorgt hat.

Open Access Dieses Kapitel wird unter der Creative Commons Namensnennung 4.0 International Lizenz (http://creativecommons.org/licenses/by/4.0/deed.de) veröffentlicht, welche die Nutzung, Vervielfältigung, Bearbeitung, Verbreitung und Wiedergabe in jeglichem Medium und Format erlaubt, sofern Sie den/die ursprünglichen Autor(en) und die Quelle ordnungsgemäß nennen, einen Link zur Creative Commons Lizenz beifügen und angeben, ob Änderungen vorgenommen wurden.

Die in diesem Kapitel enthaltenen Bilder und sonstiges Drittmaterial unterliegen ebenfalls der genannten Creative Commons Lizenz, sofern sich aus der Abbildungslegende nichts anderes ergibt. Sofern das betreffende Material nicht unter der genannten Creative Commons Lizenz steht und die betreffende Handlung nicht nach gesetzlichen Vorschriften erlaubt ist, ist für die oben aufgeführten Weiterverwendungen des Materials die Einwilligung des jeweiligen Rechteinhabers einzuholen.

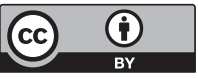

